\title{
Uso de las tecnologías de la información y la comunicación (TIC) como factor de competitividad en la gestión del sector hotelero en Villavicencio, Colombia
}

DOI: https://doi.org/10.21158/01208160.n0.2020.2739

Fecha de recepción: 25 de septiembre de 2019

Fecha de aprobación: 22 de octubre de 2020

Fecha de publicación: 03 de diciembre de 2020
Hugo Alberto Martínez-Jaramillo ${ }^{1}$

Universidad Nacional Abierta y a Distancia-UNAD

hugo.martinez@unad.edu.co

Johana Moreno-Duarte ${ }^{2}$

Universidad Nacional Abierta y a Distancia-UNAD johanamorenoduarte@hotmail.com

Cómo citar este artículo / To reference this article / Comment citer cet article / Para citar este artigo:

Martínez-Jaramillo, H. A.; Moreno-Duarte, J. (2020). Uso de las tecnologías de la información y la comunicación (TIC) como factor de competitividad en la gestión del sector hotelero en Villavicencio, Colombia. Revista Escuela de Administración de Negocios, Edición especial 2020, 93 - 114. DOI: https://doi.org/10.21158/01208160.n0.2020.2739

\section{Resumen}

El objetivo de este artículo es analizar el uso de las tecnologías de la información y la comunicación (TIC) en la gestión del sector hotelero en Villavicencio, Colombia. El estudio es de tipo descriptivo y aplica encuestas semiestructuradas. Se tomó como población objetivo 34 hoteles afiliados a la Asociación Hotelera y Turística de Colombia (Cotelco), Capítulo Meta. El instrumento constó de 31 preguntas. Los análisis factoriales realizados al instrumento establecieron siete dimensiones principales: caracterización, infraestructura tecnológica, conectividad, frecuencia de ingreso a internet, usabilidad de las TIC en la gestión hotelera, apropiación de las TIC para la gestión del hotel y contribución de las TIC a la gestión del hotel. El instrumento arrojó un alfa de Cronbach del 0,921, de modo que mostró una alta fiabilidad en los ítems relacionados en cada una de las dimensiones. El análisis hizo posible identificar las TIC que más impacto tienen en la gestión hotelera, así como los aspectos que se deben mejorar, con el fin de consolidar la transformación digital del sector. Se concluye, entonces, que es necesario que sector hotelero en Villavicencio maximice su capacidad instalada en términos de infraestructura tecnológica, aprovechando el potencial que se tiene en la región en términos de oferta turística, en la cual el sector hotelero es un actor relevante dentro de estas dinámicas sectoriales.

Palabras clave: transformación digital; sector hotelero; gestión hotelera; competitividad hotelera; infraestructura tecnológica; tecnologías de la información y la comunicación (TIC).

\footnotetext{
${ }^{1}$ MBA. UNAD Florida (Estados Unidos). Especialista en Gerencia Estratégica de Mercadeo. Universidad Nacional Abierta y a Distancia-UNAD. Economista Universidad Católica de Colombia. ORCID: https://orcid.org/0000-0001-8190-9993

2 Estudiante Especialización en Gerencia Estratégica de Mercadeo. Universidad Nacional Abierta y a Distancia - UNAD. Administradora de Empresas, Universidad Nacional Abierta y a Distancia -UNAD. Zona Amazonia y Orinoquia. ORCID: https: / / orcid.org/0000-0003-4190-0534
} 


\title{
Using information and communication technologies (ICT) as a competitive factor in the management of the hotel sector in Villavicencio, Colombia
}

\begin{abstract}
The aim of this article is to analyze the use of information and communication technologies (ICT) in the management of the hotel sector in Villavicencio, Colombia. The study is descriptive and applies semi-structured surveys. The target population was 34 hotels affiliated to the Hotel and Tourism Association of Colombia (Cotelco), Chapter: Meta. The instrument comprised 31 questions. The factor analyses carried out on the instrument established seven crucial dimensions: characterization, technological infrastructure, connectivity, frequency of internet use, usability of ICTs in hotel management, appropriation of ICTs for hotel management, and contribution of ICTs to hotel management. The instrument yielded a Cronbach's alpha of 0.921, showing high reliability in the related items in each of the dimensions. The analysis made it possible to identify the ICTs that have the greatest impact on hotel management, and the aspects to be improved in order to consolidate the digital transformation of the sector. The conclusion must then be that it is necessary for the hotel sector in Villavicencio to maximize its installed capacity in terms of technological infrastructure, taking advantage of the potential that the region has in terms of tourism supply, in which the hotel sector is a relevant actor within these sector dynamics.
\end{abstract}

Keywords: digital transformation; hotel sector; hotel management; hotel competitiveness; technological infrastructure; information and communication technologies (ICT).

\section{Uso das tecnologias de informação e comunicação (TIC) como fator competitivo na gestão do setor hoteleiro em Villavicencio, Colômbia}

\section{Resumo}

O objetivo deste artigo é analisar o uso das tecnologias de informação e comunicação (TIC) na gestão do setor hoteleiro em Villavicencio, Colômbia. O estudo é de caráter descritivo e aplica questionários semiestruturados. A população-alvo foram 34 hotéis filiados à Associação de Hotelaria e Turismo da Colômbia (Cotelco), Capítulo Meta. $\mathrm{O}$ instrumento era composto por 31 questões. A análise fatorial realizada no instrumento estabeleceu sete dimensões principais: caracterização, infraestrutura tecnológica, conectividade, frequência de acesso à Internet, usabilidade das TIC na gestão hoteleira, apropriação das TIC para a gestão do hotel e contribuição das TIC para a gestão do hotel. O instrumento gerou um alfa de Cronbach de 0,921, de forma que apresentou uma alta confiabilidade nos itens relacionados em cada uma das dimensões. A análise permitiu identificar as TIC com maior impacto na gestão hoteleira, bem como os aspectos que devem ser melhorados, de forma a consolidar a transformação digital do setor. Conclui-se, então, que é necessário que o setor hoteleiro de Villavicencio maximize a sua capacidade instalada em termos de infraestrutura tecnológica, aproveitando o potencial que a região possui em termos de oferta turística, onde o setor hoteleiro é um ator relevante dentro dessas dinâmicas setoriais.

Palavras-chave: transformação digital; setor hoteleiro; gestão hoteleira; competitividade hoteleira; Infraestrutura tecnológica; tecnologias de informação e comunicação (TIC). 


\section{Utilisation des technologies de l'information} et de la communication (TIC) comme facteur de compétitivité pour la gestion du secteur hôtelier de Villavicencio, Colombie

\section{Résumé}

L'objectif de cet article est d'analyser l'utilisation des technologies de l'information et de la communication (TIC) dans la gestion du secteur hôtelier de la ville colombienne de Villavicencio. Cette étude descriptive utilisera plusieurs enquêtes semi-structurées dont la cible comprend un ensemble de 34 hôtels affiliés à l'Association colombienne de l'hôtellerie et du tourisme - Cotelco-. Les analyses factorielles réalisées grâce aux 31 questions de cette investigation ont pu établir sept vecteurs principaux: la caractérisation, les infrastructures technologiques, la connectivité, la fréquence d'accès à Internet, l'utilisation, l'appropriation et la contribution des TIC dans la gestion hôtelière. Ces instruments d'analyse projettent un alpha de Cronbach de 0,921 reflétant une grande fiabilité des éléments connexes dans chacune des dimensions de l'analyse et permettent d'identifier les TIC ayant le plus d'impact sur la gestion hôtelière et ses aspects à améliorer afin de consolider la transformation numérique du secteur. Nous concluerons que le secteur hôtelier de Villavicencio doit maximiser ses capacités en termes d'infrastructures technologiques et tirer parti du potentiel touristique de la région dans lequel il est un acteur prédominant des dynamiques sectorielles.

Mots-clés: transformation numérique; secteur hôtelier; gestion hôteliére; compétitivité hôtelière; infrastructures technologiques; technologies de l'information et de la communication (TIC). 


\section{Introducción}

$\mathrm{U}^{\prime}$ n referente de cambio en la sociedad contemporánea es la presencia de las tecnologías de la información y las comunicaciones (TIC) en múltiples ámbitos y su constante evolución, dado que están presentes en todas las actividades cotidianas e inciden en la forma en la que las personas se relacionan con la información y con otras personas (De Pablos Pons, 2018; García-Peñalvo y SeoanePardo, 2015).

La globalización implica el funcionamiento de los mercados las 24 horas del día, los siete días a la semana, y conlleva la necesidad de operar desde cualquier parte del mundo a cualquier hora, lo que supone un cambio en los patrones de consumo (Oliveros y Martínez, 2017, p. 17). Así mismo, tal y como plantea Martínez (2013), «las tecnologías de la información y la comunicación tienen un papel fundamental en el incremento de la competitividad, la mejora de la productividad y el incremento de la satisfacción de los clientes».

Estas tecnologías de la información (TI) han transformado las actividades económicas en los diferentes sectores y, desde luego, el hotelero no es ajeno a esta transformación. De esta manera, el negocio hotelero tradicional se ve amenazado por una serie de factores que producen hoy un cambio profundo en el sector (Camisón, 1995). En este sentido, hoy en día, las TIC han contribuido a transformar las dinámicas organizacionales en los diferentes subsectores turísticos, entre ellos la industria hotelera.

Estos actores juegan un papel determinante en las relaciones empresariales y en la forma de aplicar la filosofía de orientación al cliente. De igual forma, es importante entender que hoy el mundo se mueve a partir de la cuarta revolución industrial. Los diferentes sectores de la economía, así como las organizaciones, en su conjunto, que dinamizan estos sectores, se ven abocados a incorporar elementos de esta cuarta revolución, teniendo en cuenta la digitalización, la cual es transversal a las diferentes actividades económicas (Schwab, 2016).

Estudios de MacFarlan (1985) y Millar y Porter (1986), como se citan en Oliveros y Martínez (2017), sugieren que las tecnologías contribuyen a una mayor competitividad de las organizaciones que saben obtener beneficio de su uso, y así agregan valor a sus procesos internos y a sus productos.

Al constituirse el sector turístico como potencial para el desarrollo productivo de la sociedad, las empresas del sector se ven supeditadas a cambios constantes generados, en gran medida, por internet y su evolución. Desde sus inicios internet ha sido revolucionario, dado que sugiere la comercialización a través de nuevos canales de distribución, como, por ejemplo, las agencias de viaje online - OTA, por sus siglas en inglés-, de manera que dispone para los clientes y las empresas herramientas de comunicación con capacidad de acceso global. Así, gracias a internet surge una posibilidad para el turismo y la reingeniería de procesos de negocio, impulsada por las TIC observadas en la industria, genera, de modo gradual, un nuevo cambio de paradigma (Buhalis y Law, 2008, p. 610).

Internet, en la actualidad, es una herramienta de gran relevancia para la comercialización de productos y servicios, además de que puede contribuir a mejorar la eficiencia y la competitividad tanto de las organizaciones como de diversos sectores. Adicionalmente, presenta ciertas ventajas, tales como mayor cobertura geográfica, acceso a mercados globales y multicanalidad en la comunicación aplicaciones de mensajería, redes sociales, etc.-; a diferencia de otros medios, como, por ejemplo, la televisión, la prensa o la radio, entre otros.

La adopción de internet en cualquier industria ocurre por determinados factores que la favorecen. La industria turística, en particular, posee diferenciales que hacen que sea beneficioso adoptar internet 
como medio de comunicación y comercialización (Geyskens, Steenkamp y Kumar, 1999). Estos diferenciales pueden dinamizarse a partir de las aportaciones a la innovación.

Desde el punto de vista de la demanda, en respuesta a la gran conectividad e influencia de los turistas, se manifiestan de forma disruptiva con las posibilidades de internet el análisis del big data y el desarrollo de la inteligencia artificial. En este marco, la transformación de los destinos turísticos podría venir de la mano del paradigma de los destinos turísticos inteligentes (DTI) (Fernández-Alcantud et al., 2017, p. 138). Para algunas industrias, como, por ejemplo, la financiera, la de transporte aéreo y la de libros y discos compactos, internet ha tenido una importancia crucial.

La creación y el desarrollo de empresas virtuales interconectadas encuentran el escenario propicio en el mercado turístico (Palmer y McCole, 2000), debido a que la industria turística tiene un mercado de carácter global; por lo cual se requiere integrar ofertas locales con demandas internacionales y las TIC ofrecen un gran potencial para este tipo de desarrollos. Además, las tecnologías permiten dinamizar campañas a través de estrategias de marketing digital, posicionamiento web y demás recursos relacionados con estos avances.

La tradicionalidad hotelera está amenazada por diversos factores que llevan al sector a experimentar cambios radicales en el área tecnológica, frente a la alfabetización tecnológica de los clientes, la segmentación del mercado y la libre acción de los sistemas de distribución. Esto, sin lugar a duda, conlleva al inicio del proceso de desintermediación (Camisón,1995; Stamboulis y Skayannisb, 2003), con base en la oferta directa del sector hotelero a través de diversos medios digitales, tales como redes sociales —Facebook, Instagram, Twitter-, videos en Youtube o e-mail marketing, entre otros recursos.

Estos nuevos mecanismos de distribución contribuyen a evitar las prácticas de oligopolio de los operadores turísticos, así como mejoran la competitividad y las posibilidades de desarrollo para las pequeñas y medianas industrias (Bastakis, Buhalis y Butler, 2004), dado que dentro de las ventajas que ofrecen las TIC podemos mencionar, entre otras, el acceso a ellas por parte de todo tipo de empresas, sin tener en cuenta el tamaño de la organización - micro, mediana, pequeña o gran empresa-

Paralelo al desarrollo de las TIC se generan nuevas necesidades entre los consumidores, y el hecho de no atenderlas implicará una disminución en la calidad de los hoteles, pues deberán atender los cambios del entorno y crear una mentalidad de adaptación a ellos. Por tanto, el constante cuestionamiento sobre las necesidades de los clientes debería ser un proceso que se desarrolle de forma permanente en la estrategia del hotel (Santomá, 2004), más aun entendiendo la dinámica de los mercados actuales, y la posibilidad que tienen los consumidores de buscar alternativas de manera ágil y oportuna a través de las TIC. Es por esto que se resalta la necesidad de que el sector entienda estas dinámicas y esté en capacidad de adaptar su oferta de acuerdo con las necesidades y las expectativas del mercado.

Según Oliveros y Martínez (2017), las aplicaciones más relevantes de las TIC en el sector turístico las utilizan las agencias de viajes, las aerolíneas, los restaurantes, los proveedores y los hoteles. Estas han sido encaminadas, principalmente, hacia la implementación de sistemas de reservas en línea, ofertas, promociones y ventas de servicios por internet, así como hacia la interacción y la respuesta rápida a las necesidades del cliente, entre otros; alternativas que afectan no solo la producción del servicio, sino también su gestión y distribución (Alberca y Parte, 2010).

Así mismo, para Botello, Pedraza y Contreras (2015), toda la información que las empresas poseen de los clientes, productos y proveedores es su recurso más valioso, información que puede capturarse a través de diversas aplicaciones, con lo cual se puede conocer de manera más concreta las tendencias y los gustos de los consumidores. La implementación de las TIC y su introducción dentro de los procesos de producción es clave para el incremento de su competitividad, pues se convierten en las mejores herramientas que tienen las empresas para enfrentar este contexto. En este 
sentido, el uso que se dé a la información, teniendo en cuenta, desde luego, la normatividad del país en cuanto a la ley de protección de datos, es vital para que las TIC generen un mayor valor y que esto redunde en una mayor eficiencia y competitividad del sector.

Un compendio de las principales TIC aplicadas en hoteles incluye la dotación de hardware y software, las tecnologías de conexión a redes, los procesos de negocio integrados, las aplicaciones de marketing digital y de ventas, así como los sistemas de información destinados a la gestión de las relaciones con los clientes y los proveedores (Ruiz, Gil y Moliner, 2012).

En un análisis microeconómico adelantado por Bresnahan, Brynjolfsson y Hitt (2002), estos autores encuentran que la adopción de TIC en la empresa viabiliza cambios en su interior que inciden de manera positiva sobre la calidad y permiten reducir costes. Esta reducción de costos se deriva de la automatización de ciertos procesos, la reducción de infraestructura en tecnología y una mayor cobertura de mercado, entre otros factores.

Jacob y Aguiló (2008) afirman que complementarios a los modelos de gestión tradicionales de capacidad de carga se ha innovado con el desarrollo de tecnologías que, además de simplificar cálculos y optimizar la obtención y el análisis de datos, permiten incrementar las tasas de ocupación con tácticas como el overbooking o la subasta de habitaciones y asientos disponibles. De esta manera accionan realmente en lo que se refiere a la gestión de ingresos, que ha sido especialmente implementada en hoteles, con miras, predominantemente, al precio y la duración de la estancia.

Choi y Mattila (2006), así como Lindenmeier y Tscheulin (2008), remarcan la importancia de la gestión de ingresos - revenue management- o diferentes políticas de discriminación de precios, básicas para la optimización de la capacidad, los ingresos $y$, en general, la calidad. Desde esta perspectiva, a fin de optimizar esta estrategia, es necesario incorporar todo el concepto de análisis de datos, partiendo de la captura de información, por medio de diferentes aplicaciones y recursos —redes sociales, por ejemplo-, con lo cual se tengan los elementos necesarios para focalizar la estrategia $y$ vender el servicio adecuado al cliente adecuado.

En Colombia el turismo es un sector emergente con enorme potencial de crecimiento, dadas las bondades ambientales y socioeconómicas del país. Además, cuenta con una abundante riqueza natural y cultural apta para el turismo sostenible. Las estrategias que permitan convertir este patrimonio en productos de clase mundial aún están en construcción; una de ellas es el modelo de negocio basado en las TIC que ayudará a las mipymes a mejorar su competitividad, así como les permitirá consolidar los destinos turísticos y darlos a conocer a nivel internacional (López y López, 2018).

El sector turismo en el departamento del Meta se visualiza como uno de los sectores con mayor potencial para jalonar el desarrollo. En el Plan Regional de Competitividad del Departamento del Meta 2008-2032, se contempló como un eje estratégico, al plantear lo siguiente:

El turismo y la oferta alimentaria orientada al mercado interno -andino-, son renglones promisorios en el mercado interno. Igualmente, el Meta deberá fortalecer su liderazgo en logística, servicios de salud, educación y proveedor de servicios empresariales para la región de la Orinoquía. (Gobernación del Meta, 2009, p. 26)

Así mismo, en el Plan de Desarrollo del Meta 20162019, «Tierra de oportunidades», se relacionó el «programa 1, competitividad turística: la oportunidad del Meta», con lo cual se busca incentivar, dinamizar y fortalecer el sector. Desde esta perspectiva, las TIC incorporadas a la gestión del sector hotelero deben contribuir a dinamizar el turismo en la región, dado el alto potencial que existe. 


\section{Metodología}

T a investigación fue de tipo descriptivo, aplicando Lencuestas semiestructuradas en los hoteles afiliados a Cotelco en Villavicencio y que hicieron parte de la muestra, con el fin de dar respuesta a las variables planteadas. Así mismo, se utilizó el software SPSS para el análisis de los resultados.

El instrumento constó de 31 preguntas. Los análisis factoriales realizados al instrumento establecieron siete dimensiones principales: caracterización, infraestructura tecnológica, conectividad, frecuencia de ingreso a internet, usabilidad de las TIC en la gestión hotelera, apropiación de las TIC para la gestión del hotel y contribución de las TIC a la gestión del hotel. El instrumento arrojó un alfa de Cronbach del 0,921, mostrando una alta fiabilidad en los ítems relacionados en cada una de las dimensiones.

Se tomó como población objetivo 34 hoteles afiliados a Cotelco, Capítulo Meta, ubicados en Villavicencio, Meta, Colombia. Esta muestra se justifica dado que, en el momento del estudio, estos eran los hoteles afiliados a esta asociación.

\section{Análisis de los resultados}

Tabla 1. Dimensiones y categorías de clasificación

\begin{tabular}{|l|l|}
\hline \multicolumn{1}{|c|}{ Dimensión } & \multicolumn{1}{c|}{ Característica } \\
\hline Caracterización & $\begin{array}{l}\text { En esta dimensión se analizan aspectos concernientes a la } \\
\text { antigüedad del hotel, el estrato socioeconómico, la capacidad } \\
\text { instalada y la vocación turística. }\end{array}$ \\
\hline Infraestructura tecnológica & Tipo de infraestructura en hardware y software de los hoteles. \\
\hline Conectividad & Tipo de conectividad con la que cuentan los hoteles. \\
\hline Frecuencia de ingreso a internet & $\begin{array}{l}\text { Nivel de frecuencia de acceso para realizar gestión en los } \\
\text { hoteles. }\end{array}$ \\
\hline Usabilidad de las TIC en la gestión hotelera & Uso de las TIC para la gestión de los hoteles. \\
\hline Apropiación de las TIC para la gestión hotelera & $\begin{array}{l}\text { Grado de apropiación de las TIC para la gestión hotelera, por } \\
\text { parte del talento humano del sector. }\end{array}$ \\
\hline Contribución de las TIC a la gestión hotelera & $\begin{array}{l}\text { Contribución de las TIC en la gestión del hotel y la } \\
\text { competitividad y mejoramiento de ingresos del sector. }\end{array}$ \\
\hline
\end{tabular}

Fuente. Elaboración propia. 


\subsection{Caracterización}

Figura 1. Antigüedad en el mercado

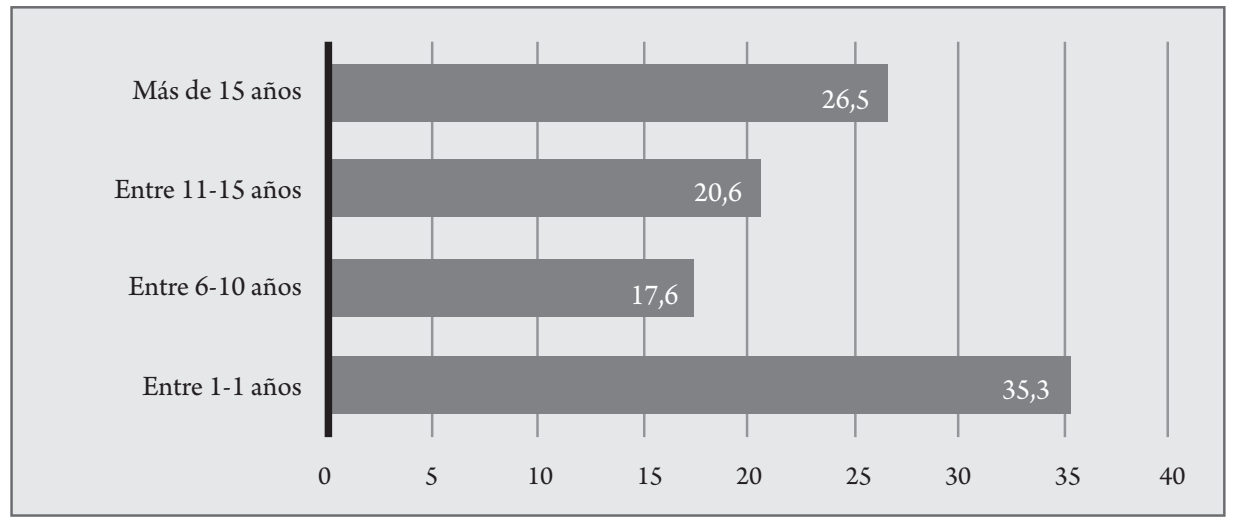

Fuente. Elaboración propia.

Un aspecto importante en la oferta hotelera de una ciudad, región o país es la capacidad instalada con la que cuenta en términos de infraestructura física. En este sentido, la antigüedad de los hoteles en Villavicencio (véase la Figura 1) se encuentra, principalmente, entre uno y cinco años, con un $35,3 \%$, mientras que los hoteles más antiguos, con más de 15 años, tienen una participación del 26,5 \%. En menor proporción, se encuentran entre 11 y 15 años, con un $20,6 \%$, y entre seis y diez años con un $17,6 \%$.
Esta variable indica que la infraestructura hotelera en Villavicencio es relativamente nueva, con lo cual se tiene una oferta en condiciones favorables que permite dar respuesta a las expectativas del cliente. Este factor, sumado al de la gestión del sector hotelero a través de las TIC, permite que exista una oferta integral en cuanto a los servicios turísticos.

Figura 2. Estrato socieconómico en el que se encuentra ubicado el hotel

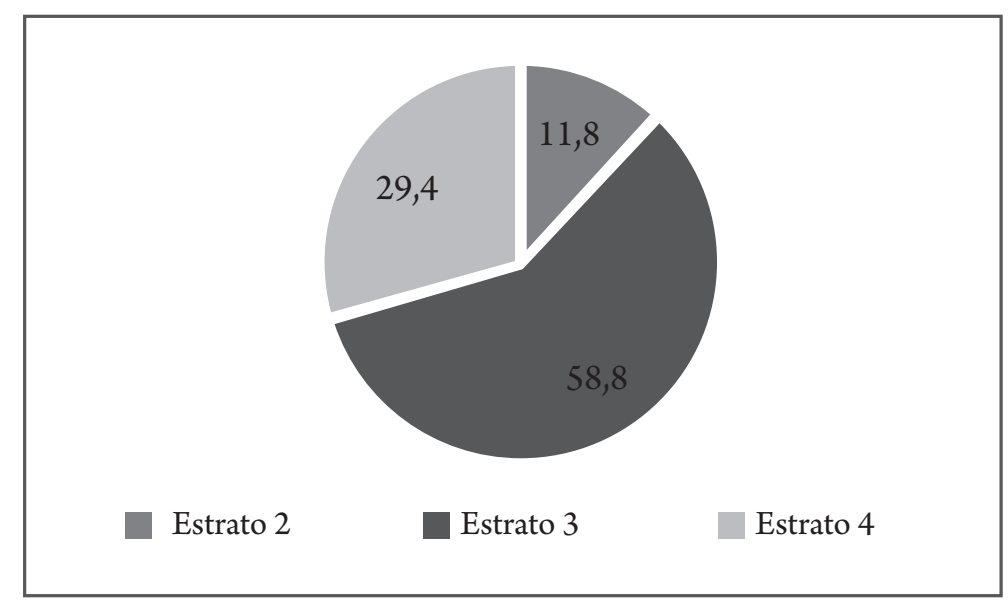

Fuente. Elaboración propia. 
En lo referente a la ubicación de los hoteles (Figura 2), la mayor parte se encuentra ubicada en el estrato tres, con un $58,8 \%$, y en el estrato 4 , con un $29,4 \%$. En menor proporción en el estrato dos, con un $11,8 \%$. En Colombia la estratificación socioeconómica es una clasificación en estratos de los inmuebles que deben recibir servicios públicos. En este orden de ideas, al estar ubicada la mayor parte de los hoteles - 84,2 \% - en los estratos 3 y 4, existe mayor probabilidad de acceder a los servicios públicos y, en este caso particular, al servicio de internet, uno de los factores clave para la incorporación de las TIC a la gestión del sector.

Con respecto al número de plazas de los hoteles, el $50 \%$ tiene entre una y 50 plazas, es decir, que se pueden clasificar como pequeños hoteles, seguido de un $23,5 \%$ con hoteles que tienen entre 51 y 100 plazas. El 14,7 \% de los hoteles tienen entre 101 y 150 plazas y $8,8 \%$ más de 200 plazas.

En lo que respecta a las habitaciones, los hoteles cuentan con la siguiente capacidad: el 41,2 \% tiene entre una y 25 habitaciones, y solo el 5,9\% cuenta con más de 75 habitaciones. Esto implica que, a pesar de que la ciudad cuenta con una infraestructura relativamente nueva, no se han hecho inversiones altas en cuanto a construcción de hoteles con gran capacidad. Esto, por ser una ciudad intermedia que, según el Censo 2018, cuenta con 492052 habitantes en su cabecera municipal. Sin embargo, se debe tener en cuenta que se encuentra, aproximadamente, a 115 kilómetros de Bogotá, ciudad capital de Colombia, con 7387400 habitantes, lo cual ofrece un potencial turístico para la región.

Sobre lo relacionado con la vocación hotelera, el $61,8 \%$ tiene vocación mixta, el $26,5 \%$ corporativa y el $11,8 \%$ vacacional. Es importante destacar que la mayor parte de los hoteles tienen la capacidad de dar respuesta a los clientes que buscan actividades de tipo corporativo y vacacional. En lo referente a lo vacacional, el porcentaje es bajo si se tiene en cuenta el potencial turístico de la región en cuanto a clima, folclor, cultura y gastronomía, entre otros aspectos, además de su cercanía con Bogotá.
Sobre el tema corporativo, Villavicencio es la entrada al Llano, como se conoce por su ubicación geográfica, dado que es el paso obligado para ir a los municipios que se encuentran más hacia la llanura, como, por ejemplo, Puerto López, Puerto Gaitán, Acacías, Granada, Guamal y San Martín, entre otros. En este sentido, por estar varios de estos municipios relacionados con la industria petrolera, empleados de este sector se ubican en los hoteles de Villavicencio, ya que al ser la ciudad más grande en la región cuenta con una oferta variada de bienes y servicios, atributos atractivos para el alojamiento.

\subsection{Infraestructura tecnológica}

Dado que uno de los aspectos importantes para la gestión organizacional es lo referente a la infraestructura tecnológica, bien sea propia o bien tercerizada - computación en la nube-, el sector hotelero en Villavicencio cuenta con servidores de almacenamiento de marcas reconocidas, como, por ejemplo, HP, en una mayor proporción con un $29,4 \%$; de igual manera, hace uso de servidores genéricos en un 20,6\% y, en menor proporción, de otras marcas como Lenovo, Dell, LG, Movistar y Panasonic.

Con respecto al sistema operativo de los servidores, la mayor parte utiliza Windows, con un $76,5 \%$, y en menor proporción Linux, con el 8,8 \%. Los demás hoteles no utilizan ningún sistema operativo, dado que, por ser pequeños hoteles, no cuenta con infraestructura tecnológica tipo servidor, sino que manejan la información en computadores personales, ya sean de escritorio o portátiles; en algunos casos más puntuales, parte de la gestión administrativa se maneja mediante libros contables.

En lo que tiene que ver con el uso de router, la mayor parte de hoteles utiliza la marca TP-Link con un $35,3 \%$, seguida de Huawei con un $14,7 \%$, y en menor proporción marcas como Cisco, Asus y HP. Con respecto a los acces point, el 29,4\% utiliza TP- Link, el 17,6 \% HP, Huawei el 8,8 \% y Ubiquiti el 5,9\%. Es importante resaltar este aspecto, dado que la disponibilidad de internet para los usuarios o clientes 
de los hoteles depende, en gran medida, de la calidad y las características de estos dispositivos.

En cuanto a impresoras, el uso en su mayor parte se concentra en las marcas HP y Samsung, con un 32,4\% y un 20,6\%, respectivamente, seguidas de la marca Cannon con un $17,6 \%$. Otro tipo de impresoras, como, por ejemplo, Epson, Kyocera y Brother se usan en menor proporción. Con respecto a los equipos de escritorio, la marca más usada es Lenovo, con un $41,2 \%$, seguida de HP, con un $32,4 \%$. Con un menor uso se encuentran los equipos genéricos con un $14,7 \%$ y Asus con un 5,9\%.

En lo referente al uso de portátiles, la marca de mayor uso es Lenovo, con un $23,5 \%$, seguida de $\mathrm{HP}$, con un $20,6 \%$, de manera que son las marcas más utilizadas. En menor proporción se encuentra el uso de otras marcas como Dell, con un 5,9\%, así como Asus y Samsung con un 2,9\%, respectivamente. Con respecto al uso de smartphones, el 47,1 \% utiliza Samsung, el 32,4 \% Huawei, las de mayor uso, y con un menor uso Apple y LG, con el 2,9\%.

Con respecto a los tipos de seguridad electrónica, el 94,1 \% utiliza circuito cerrado de televisión, el 23,5\% centros de control de alarmas y el $8,8 \%$ lector de huella.

En cuanto a la infraestructura tecnológica relacionada con el software, el 82,4 \% utiliza software de oficina - paquetes de Office o Linux, entre otros-, el $35,3 \%$ software contable, el 8,8 \% sistemas ERP y el $2,9 \%$ CRM. En lo que tiene que ver con sistemas de comunicación interna, el 76,5 \% utiliza chat y correo electrónico, y el 35,3\% software hotelero especializado.

Figura 3. Servicios en SaaS, PaaS, IaaS



Fuente. Elaboración propia.

Sobre los servicios que actualmente ofrecen compañías a nivel mundial (véase la Figura 3) en temas como SaaS (software como servicio), PaaS (plataforma como servicio), IaaS (infraestructura como servicio), se hace muy poco uso de la analítica de datos y/o big data, con un 2,9\%, o de la seguridad informática también con un 2,9\%. En cuanto al backup como respaldo e internet de las cosas, se hace un mayor uso, pero sigue siendo bajo, con un 14,7 \% para ambos casos. El porcentaje de mayor uso es en lo referente a almacenamiento en la nube con un 20,6\%.
En lo que tiene que ver con licenciamientos, el $50 \%$ de los hoteles cuenta con software licenciado, y el $50 \%$ sin licenciar. Del $50 \%$ que tiene software licenciado, el 26,5 \% cuenta con la última versión, y el 73,5 \% con versiones anteriores. En cuanto a servicios en infraestructura tecnológica, el 8,8 \% de los hoteles cuenta con facturación electrónica, el 11,8 \% con B\&B Bed \& Breakfast online, el 35,3 \% con sistema electrónico Check in-Check out, el 23,5\% con auditoría interna y el 8,8 \% con gestión de ama de llaves - housekeeping-. 


\subsection{Conectividad}

En lo referente a la conexión a internet, el $100 \%$ del sector cuenta con conectividad a internet. Con respecto al acceso a la red por parte del cliente, el $76,5 \%$ lo hace con única clave, el $11,8 \%$ por red pública, el $8,8 \%$ por clave única por habitación y el $2,9 \%$ con código por tiempos. En este sentido, para el cliente es importante la forma en que accede a internet por temas de privacidad y seguridad de la información, como parte de la oferta en tecnología que brindan los hoteles.

En cuanto a la conectividad del hotel expresada en $\mathrm{Mb}$, el 73,5\% cuenta con una conectividad mayor a $11 \mathrm{Mb}$, y el 26,5\% entre 1 y $10 \mathrm{Mb}$. En lo referente al tipo de conexión, el $85,3 \%$ tiene banda ancha más wifi —inalámbrica一, y el 14,7 \% banda ancha —Adsl por cable-; desde esta perspectiva los clientes cuentan con una buena disponibilidad en términos de acceso a internet. Así mismo, la velocidad es importante para la gestión de servicios apoyados en TIC, tales como almacenamiento en nube, virtualización de máquinas y software como servicio, entre otros.

\subsection{Usabilidad de las TIC en la gestión hotelera}

Con respecto al uso de las herramientas TIC como apoyo a la gestión del hotel (véase la Figura 4), se observa un mayor uso de WhatsApp con un uso entre frecuente y muy frecuente del 70,6 \%, seguido del uso de los motores de búsqueda de hoteles —-Trivago, Booking, Kayak, Hoteles.com, entre otros-, con un 67,6 \%. El uso de Facebook es del 50 \%, Facebook Messenger tiene una usabilidad del 29,4 \%, Instagram 23,5 \%, Youtube 17,5 \% y Twitter $11,7 \%$.

Figura 4. Herramientas TIC como apoyo a la gestión del hotel

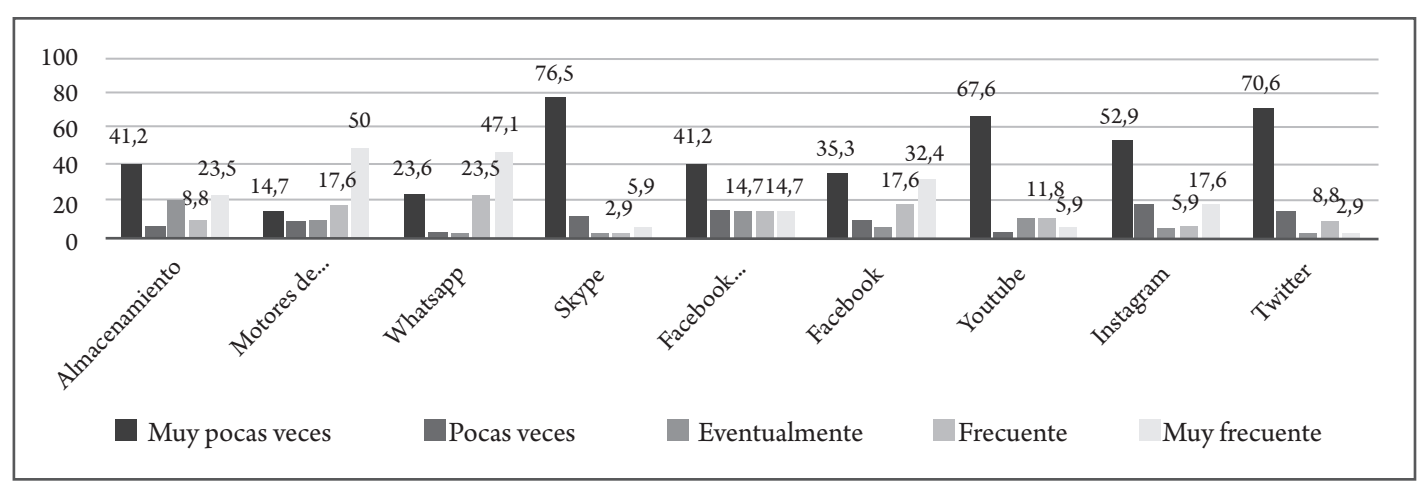

Fuente. Elaboración propia.

\subsection{Frecuencia de ingreso a Internet}

Con respecto a la usabilidad de dispositivos para acceder a Internet como apoyo a la gestión del hotel por parte de los empleados, el 70,6 \% ingresa a través del computador de escritorio, el $38,2 \%$ usa smartphone y el $26,5 \%$ lo hace a través de portátiles.

En lo que tiene que ver con los días de la semana, el lunes es el día que más ingresan los empleados para realizar actividades relacionadas con la gestión del hotel, en un $61,8 \%$, seguido del viernes con un $50 \%$, el miércoles y el jueves con un $47,1 \%$, el martes con un $44,1 \%$, y el sábado y el domingo con un $26,5 \%$, respectivamente.

En cuanto al horario de ingreso, el 44,1\% ingresa entre las 6: 00 a. m. y las 12: 00 p. m., y las 12: 00 p. m. y las 6: 00 p. m.; en una menor proporción entre las 12: 00 a. m. y las 6: 00 a. m., y entre las 6: 00 p. m. y las 12: 00 a. m., respectivamente.

El promedio de horas que ingresan es entre una y dos horas diarias, con un 29,4\%, y entre tres y cuatro horas con 26,5 \%. En menor proporción, menos de una hora, con un 5,9\%, y entre seis y siete horas, con un $2,9 \%$. 
Sobre el uso de herramientas para asesoría en línea o servicio al cliente (véase la Figura 5), el mayor porcentaje de empleados en los hoteles utiliza el celular con un 73,5\%, seguido de la asesoría de manera personal con un 70,6\%; así mismo, el teléfono fijo es aún muy utilizado con un $58,8 \%$. En menor proporción se utiliza el chat virtual en páginas web y el Facebook Messenger con un porcentaje de uso del $8,8 \%$ cada uno.

Figura 5. Herramientas de asesoría en línea y/o servicio al cliente.

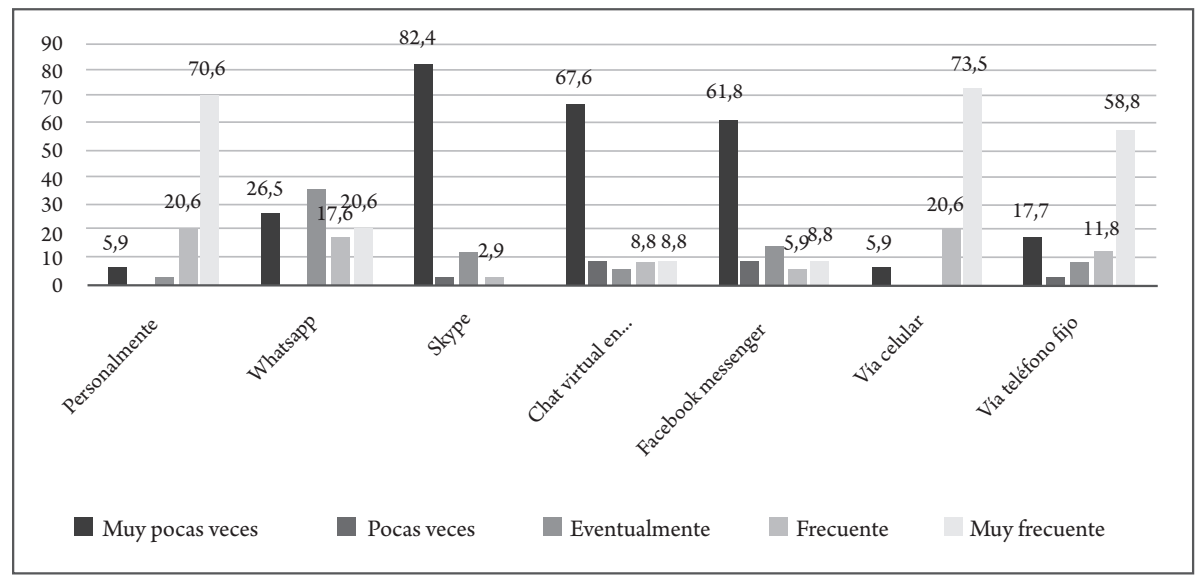

Fuente. elaboración propia.

En cuanto a los recursos y las herramientas que ofrece el marketing digital (véase la Figura 6), el recurso más utilizado es el que tiene que ver con WhatsApp, con un uso entre frecuente y muy frecuente del $50 \%$, le sigue el uso de e-mail marketing, con un $47,1 \%$, agencias de viaje online (OTA) con un $38,2 \%$, y fanpage de Facebook con un $35,2 \%$. En menor proporción, el uso de noticias en medios digitales con un 17,7\%, Google Analytics con un $12,7 \%$, controladores de contenido con un $8,8 \%$ y Google Trends con un 2,9\%.

Figura 6. Herramientas de marketing digital



Fuente. Elaboración propia. 
En lo que respecta a la percepción sobre la importancia de las redes sociales —Facebook, Instagram, Google +, Linkedin, Twitter, entre otras - (véase la Figura 7), el 91,2 \% está entre totalmente de acuerdo y muy de acuerdo en que juegan un papel importante para la estrategia de marketing digital en el hotel.

Figura 7. Las redes sociales juegan un papel importante en la estrategia de marketing digital del hotel

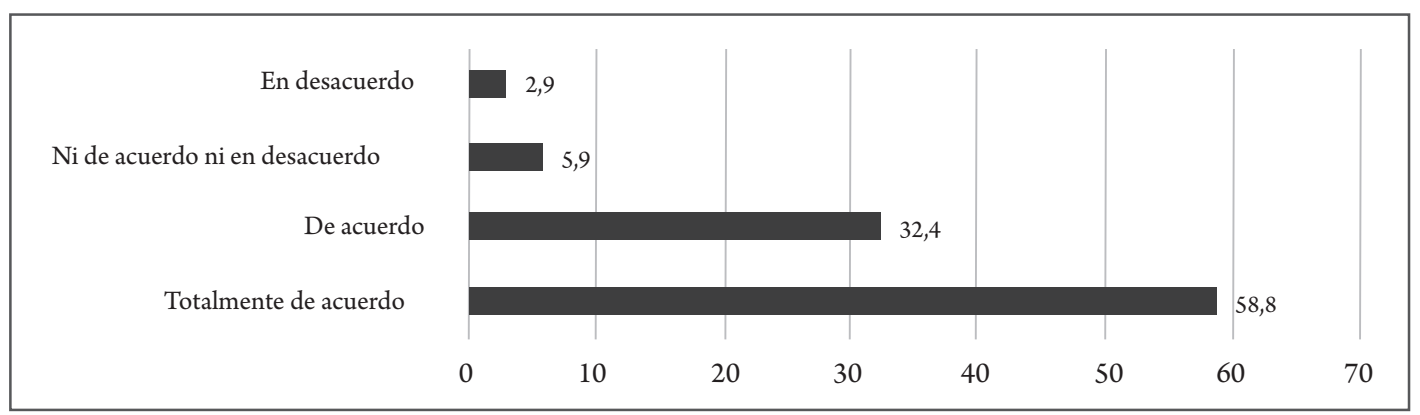

Fuente. Elaboración propía.

En lo que tiene que ver con el uso de las redes sociales para la gestión del hotel (véase la Figura 8), el 85,3 \% está entre totalmente de acuerdo y muy de acuerdo en que hacen uso de algunas de estas redes.

Figura 8. Incorporación de las redes a la gestión del hotel

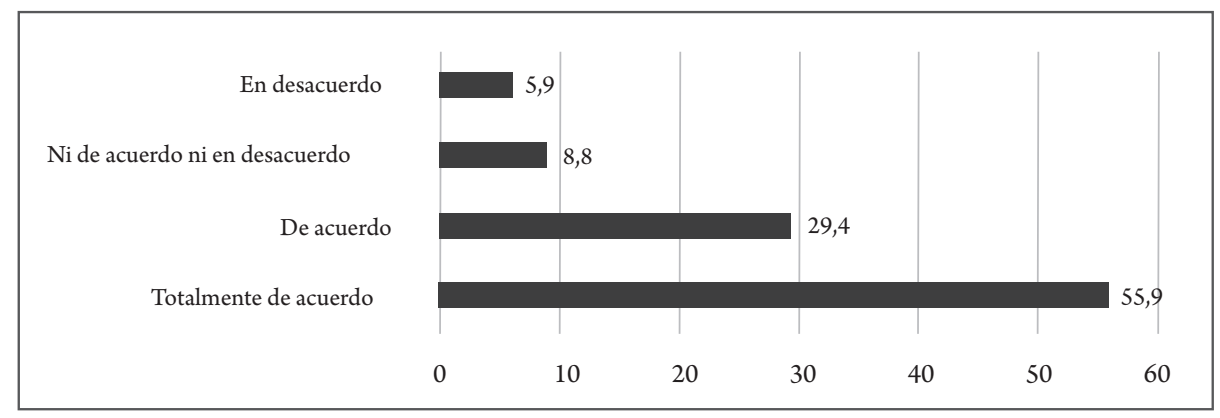

Fuente. Elaboración propia.

En cuanto a las estrategias utilizadas en las redes sociales (véase Figura 9), el 38,2 \% utiliza con más frecuencia Instagram fanpage, el 8,8 \% contenido en Youtube, y el 2,9\% Twitter, entre las más destacadas. En cuanto a otras redes sociales, el 20,6 \% las utiliza de manera frecuente, entre las cuales podemos destacar Facebook como la más usada. 
Figura 9. Estrategias utilizadas en redes sociales

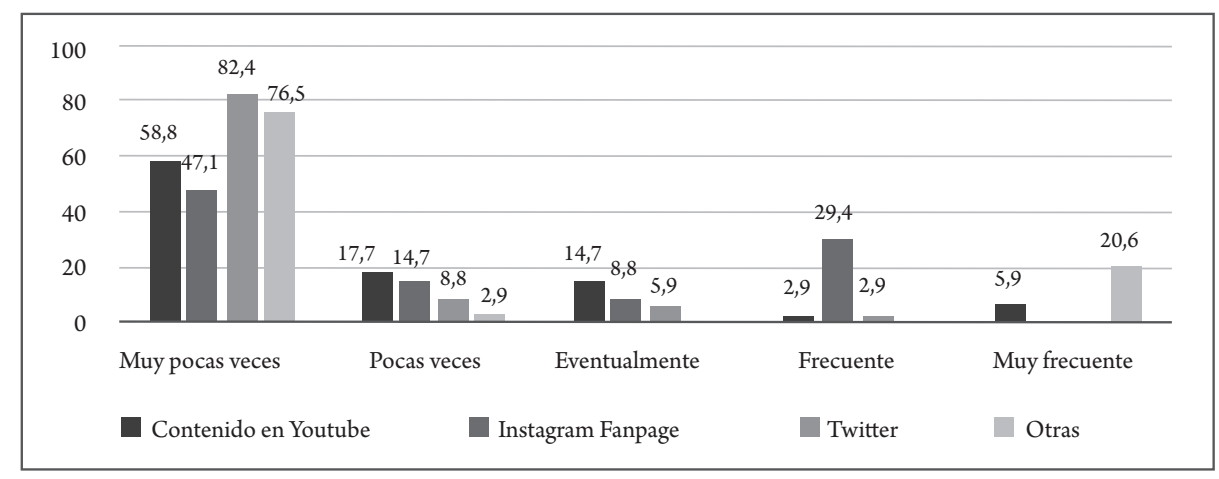

Fuente. Elaboración propia.

\subsection{Apropiación de las TIC para la gestión hotelera}

En lo que respecta al conocimiento en TIC por parte de las personas que laboran en los hoteles (véase la Figura 10), el 38,2 \% tiene conocimiento en gestión de bases de datos, y el mismo porcentaje en el uso de paquetes ofimáticos básicos. En lo que tiene que ver con gestión documental, el 29,4 \% tiene conocimiento sobre esta actividad y el 8,8 \% en programación.

Figura 10. Conocimiento en TIC por parte del talento humano de los hoteles



Fuente. Elaboración propia.

Sobre el talento humano que trabaja en las áreas de TI (véase la Figura 11), el 38,2 \% es técnico, el 26,5 \% profesional, tecnólogo el 14,7 \% y especialista el 2,9\%. En cuanto a otro tipo de conocimiento, hay un 29,4 \% referente a los contratistas, los cuales no hacen parte del hotel, pero sí prestan servicios relacionados con esta área. 
Figura 11. Personal especializado a cargo del área de TI

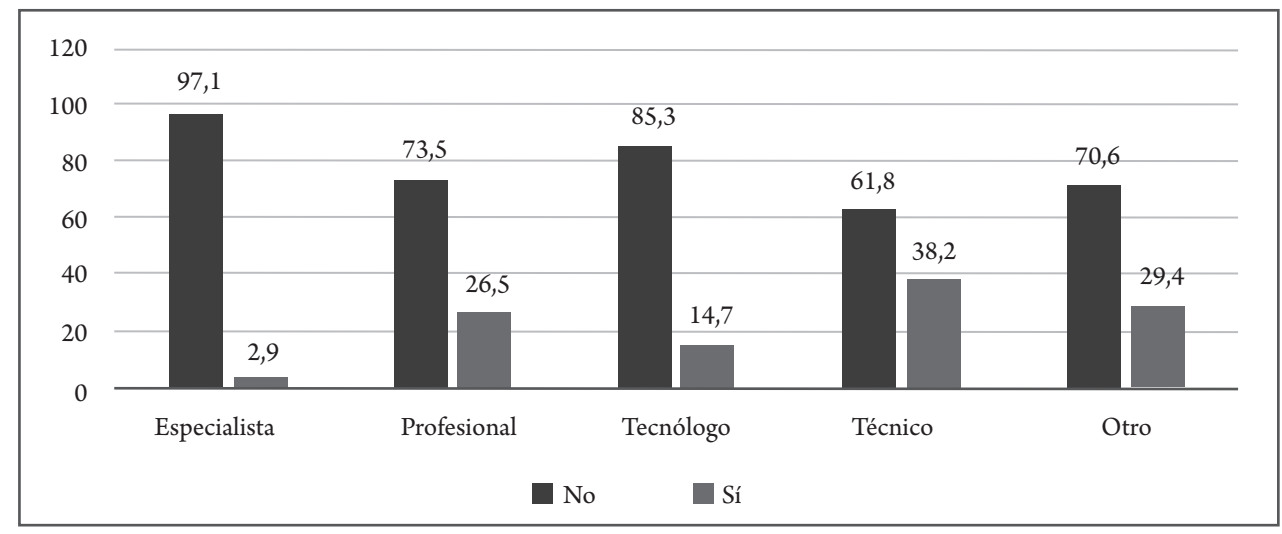

Fuente. Elaboración propia.

Con respeto a las necesidades manifiestas en cuanto a bienes y/o servicios de TI (véase la Figura 12), el 55,9 \% reconoce que existe esta necesidad dentro del sector.

Figura 12. Necesidad manifiesta en adquirir bienes o servicios de TI por parte de los hoteles

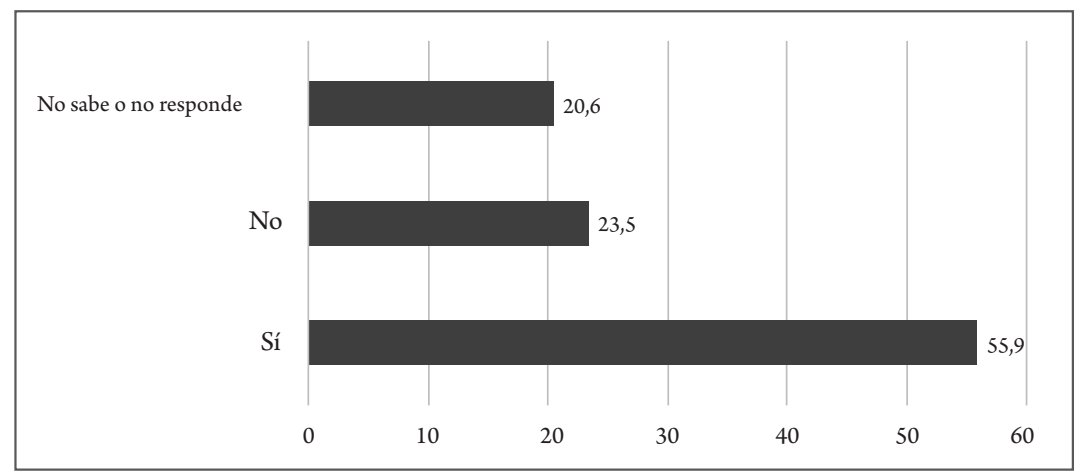

Fuente. Elaboración propia.

En cuanto a las auditorías, consultorías, asesorías o algún atipo de servicios en TI (véase la Figura 13), recibidas en los últimos años, el 52,9\% manifiesta que no ha recibido este tipo de servicios, el 26,5\% que sí, y el 20,6\% no sabe o no responde. 
Figura 13. Auditorías, consultorías y asesorías de servicios en TI recibidas por los hoteles en los últimos años

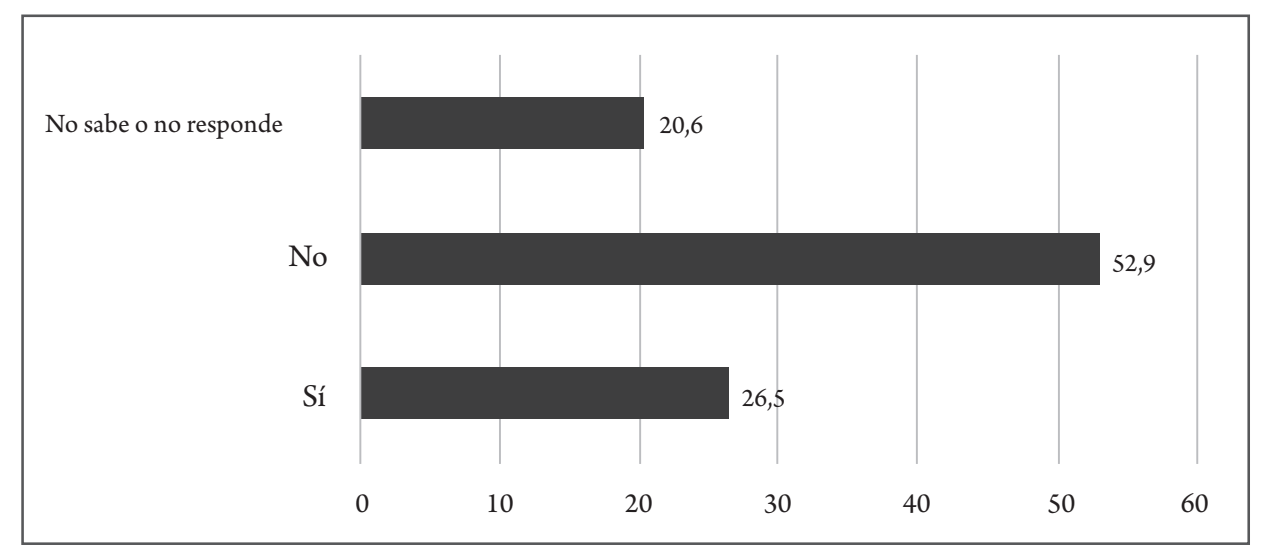

Fuente. Elaboración propia.

\subsection{Contribución de las TIC a la gestión hotelera}

Sobre el fortalecimiento de las competencias en TI (véase la Figura 14), el 91,2 \% considera que se deben fortalecer, y el 8,8\% que no es necesario hacerlo. Cabe destacar la percepción positiva que tienen los funcionarios del sector con respecto a la cualificación en temas inherentes al uso de las TIC para la gestión hotelera.

Figura 14. Fortalecimiento de las competencias de los empleados en el uso de las TIC



Fuente. Elaboración propia.

Con respecto a los recursos de TI con los que cuenta el hotel (véase la Figura 15), el $50 \%$ expresa que son suficientes para la competitividad del hotel, el $29,4 \%$ no se encuentra muy seguro de esto y el $20,6 \%$ considera que no son suficientes. 
Figura 15. Suficiencia de recursos de TI para la competitividad del sector

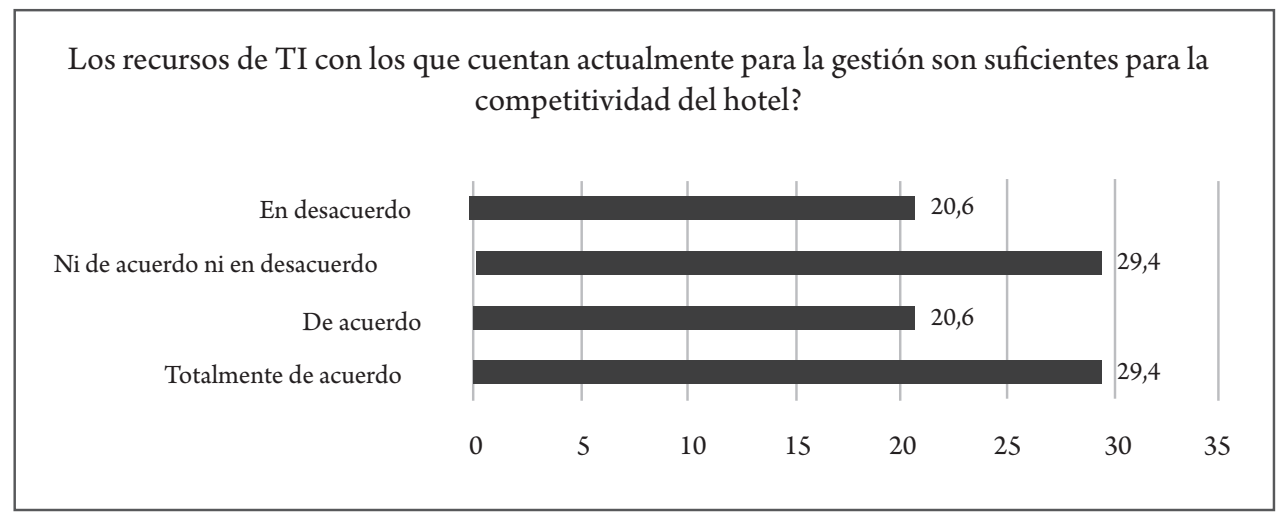

Fuente. Elaboración propia.

En lo referente a la incorporación de las TIC a la gestión hotelera (véase la Figura 16), EL 73,6 \% considera que ha contribuido al mejoramiento de los ingresos del hotel. El 17,6\% no está seguro de ello y el $8,8 \%$ piensa que no han contribuido. Es importante destacar que, en esta pregunta en particular, se toma como referente la percepción del sector en cuanto al incremento en sus ventas por el uso de las TIC en la dinámica de los procesos organizacionales.

Figura 16. Contribución de las TIC al mejoramiento de los ingresos del hotel

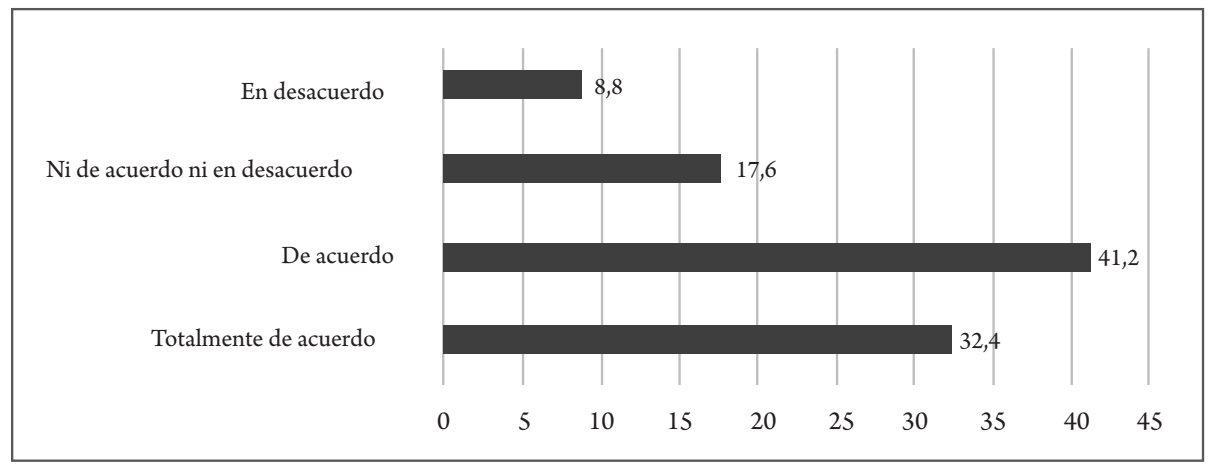

Fuente. Elaboración propia.

En cuanto a la contribución de las TIC a la competitividad del sector (véase la Figura 17), el $85,3 \%$ considera que lo hace de manera significativa, el 5,9\% no está seguro, y otro 5,9\% piensa que no contribuye de manera significativa. De igual manera, tomando como referente el análisis de la Figura 16, esta es la percepción que tienen los hoteleros en cuanto al mejoramiento de este factor, dado el uso de las TIC para la gestión. 
Figura 17. Contribución de las TIC a la competitividad del sector hotelero en Villavicencio



Fuente. Elaboración propia.

\section{Discusión de los resultados}

$\mathrm{T}_{\mathrm{e}}$ eniendo en cuenta las dimensiones de análisis, en lo referente a la caracterización de los hoteles se observa que su antigüedad se sitúa entre uno y diez años para el 52,9\%, y para el 47,1 \% en más de diez años. Esto muestra que la capacidad hotelera en Villavicencio es relativamente nueva, con lo cual se puede dar respuesta al turista, en términos de infraestructura física, con respecto al alojamiento.

Los hoteles con más de 20 años equivalen al 26,5\%. En este sentido, se destacan las inversiones en el sector, con el fin de ampliar la capacidad instalada no solo en términos de infraestructura física, sino también en infraestructura tecnológica, lo que genera las condiciones necesarias para la incorporación de las TIC a la gestión organizacional.

En lo que respecta a la estratificación, la mayor parte de los hoteles se encuentra en estrato tres, $58,8 \%$ y en estrato cuatro, $29,4 \%$, entendiendo que la estratificación económica en Colombia es una clasificación en estratos de los inmuebles que deben recibir servicios públicos. Por tanto, los sectores con mayor estratificación tienden a tener mejor cobertura en todo tipo de servicios, incluido el de redes y acceso a internet; este último es uno de los factores determinantes para la incorporación de las TIC a las dinámicas del sector hotelero.

Sobre la capacidad instalada, el 73,6 \% tiene entre una y 50 plazas, es decir, son hoteles pequeños, lo que puede dificultar de cierta manera la incorporación de las TIC a los procesos de gestión hotelera. Sin embargo, los resultados reflejan la percepción positiva que tiene el sector en su conjunto, en cuanto al uso de las TIC para las dinámicas organizacionales.

En lo relacionado con la vocación turística, el 61,8\% tiene vocación mixta, el 26,5 \% corporativa y el 11,8 \% vacacional. En este sentido, existe un potencial alto en los hoteles por atender huéspedes, tanto corporativos como vacacionales, y en menor proporción se dedican a especializarse en cierto segmento del mercado, que bien puede ser corporativo o vacacional.

En cuanto a la infraestructura tecnológica, teniendo en cuenta el tamaño de los hoteles, se centra en pequeños servidores para almacenamiento de datos, con marcas reconocidas tales como HP, Lenovo y 
Dell, entre otras. De igual manera, se usan sistemas operativos comerciales, especialmente Windows, y en menor proporción Linux. Cabe destacar que la mayor parte de los hoteles utiliza infraestructura on-premise, es decir, en el sitio físico.

De igual manera sucede con otro tipo de hardware como los acces point, los routers, las impresoras, los portátiles y los smartphones. Esta capacidad se centra en marcas reconocidas, como TP-Link en el caso de los routers y los acces-point, que es la más utilizada. En lo referente a impresoras, las marcas que más utilizan son HP y Samsung, las empresas líderes en el mercado.

Con respecto al uso de computadores tanto de escritorio como portátiles, la marca que más se usa es Lenovo, seguida de HP. Esto enfocado en la utilización de estos dispositivos para la gestión del hotel. En lo referente al uso de smartphones, se resalta el uso de Samsung, seguido por Huawei, marcas que actualmente lideran el mercado a nivel global.

De acuerdo con Jativa (como se cita en Espín, Salazar, Jara y García, 2017, p. 39),

\section{es necesario contar con aplicaciones hoteleras que permitan efectuar el curso de los negocios de forma ágil y extensible. Es innegable que los hoteles que no estén preparados para «jugar» en un terreno multiempresarial, en forma rápida y conectada, terminarán por ser desconocidos en el ámbito competitivo.}

En este sentido, la infraestructura tecnológica es suficiente para el sector, sin embargo, no existe una usabilidad adecuada con el fin de que se optimice esta capacidad para el mejoramiento de la competitividad del sector en su conjunto.

Otra variable de análisis tiene que ver con el uso de SaaS (software como servicio), PaaS (plataforma como servicio) e IaaS (infraestructura como servicio), de modo que se refleja la baja usabilidad de tecnologías relacionadas directamente con la cuarta revolución industrial, como, por ejemplo, el almacenamiento en nube, backup como respaldo y, especialmente, el poco uso de la analítica de datos y el big data, con un $2,9 \%$. Este aspecto es importante, dado que uno de los ejes principales para la transformación digital de las organizaciones y la implementación de estrategias mediadas por TIC, como, por ejemplo, el marketing digital o la dinamización de redes sociales, entre otros, que parten del conocimiento del cliente a través de los datos.

Así mismo, cabe destacar también la baja usabilidad de CRM, dada la relevancia que tiene para fortalecer las ventajas competitivas de este sector (Robledo, 1998; Zafiropoulos et al., 2006), como se citan en Padilla y Garrido (2012). En este sentido, no se están aprovechando las condiciones de conectividad para la realización, por ejemplo, de marketing relacional, como uno de los factores clave para la relación y fidelización de los clientes. En este sentido, en el sector hotelero, en el que la importancia del servicio al cliente es fundamental, se presenta especialmente la posibilidad de incorporar sistemas CRM, los cuales puede proporcionar a los hoteles numerosas oportunidades estratégicas, ya que posibilitan el apalancamiento del conocimiento de clientes (Padilla y Garrido, 2012, p. 594).

En lo referente a la conectividad, como tercera dimensión de análisis el $100 \%$ de los hoteles cuentan con conectividad, aun si se tiene en cuenta que el 14,7 \% de los hoteles no utilizan servidores y sistemas operativos para la gestión organizacional, o si tienen acceso a internet para el servicio de sus clientes y actividades personales. Del $100 \%$ que tiene conectividad, el $73 \%$ tiene una capacidad de navegación mayor a $11 \mathrm{Mb}$. Schulte, como se cita en Espín et al. (2017), sostiene que el «rápido incremento está estrechamente relacionado con el avance de la tecnología y telecomunicaciones, por lo que se ha transformado en una industria altamente globalizada». Por tanto, uno de los aspectos que se deben tener en cuenta para la incorporación y uso de las TIC es una óptima conectividad. 
De igual manera, se destacan aspectos relevantes, como, por ejemplo, la percepción positiva en un $85,3 \%$ que tienen en el sector en cuanto a la contribución al mejoramiento de la competitividad si se incorporan las TIC a la gestión hotelera. Así mismo, existe una percepción alta sobre la importancia de las redes sociales para la gestión del hotel con un $91,2 \%$.

Sin embargo, la usabilidad es baja, y si tenemos en cuenta que «conocer la ubicación de los clientes permite identificar el segmento al que este pertenece, siendo este valor necesario cuando se analiza el comportamiento de los usuarios que utilizan las redes sociales» (Madi, Brashear Alejandro y Brashear, 2016), como se citan en Majó, Moya, Vall-Llosera (2018); por lo tanto, es necesario que el sector haga un mayor uso de las redes para optimizar sus estrategias de marketing digital.

De igual manera, el 73,6 \% considera que las TIC contribuyen al mejoramiento de los ingresos. En este sentido, se deben mejorar los recursos en TI con los que cuenta actualmente el sector, dado que el $50 \%$ piensa que no son suficientes para la gestión del hotel.
Otro aspecto a destacar es el mejoramiento de las competencias en el uso de las TIC, pues el 91,2\% considera que se deben fortalecer los conocimientos en estas áreas.

Es importante tener en cuenta que el uso de las TIC en la gestión de las empresas del sector hotelero no ha alcanzado todo su protagonismo, aunque las tendencias actuales marcan pautas para lograr mayor efectividad y brindar servicios con calidad (Espín et al., 2017).

Goldhar y Jelinek, como se citan en Oliveros y Martínez (2017), afirman que las empresas que emplean tecnologías pueden obtener ventajas a través de una mayor diferenciación de productos, es decir, de productos personalizados y mejor adaptados al gusto de los clientes. Pues gracias a internet es posible ampliar el volumen de clientes, puesto que los usuarios pueden tener acceso a información privilegiada de los principales sitios turísticos, comparar ofertas, leer comentarios de otros usuarios, etc. (Oliveros y Martínez, 2017).

\section{Conclusiones}

$\mathrm{L}$ a infraestructura hotelera, en un $52 \%$, tiene entre uno y diez años, con lo cual existe capacidad instalada, relativamente nueva. En términos generales, la infraestructura tecnológica con la que cuenta actualmente el sector es acorde a las dinámicas económicas y a su capacidad.

En recursos SaaS, PaaS e IaaS, existe un bajo uso con respecto a tecnologías de la cuarta revolución industrial, como, por ejemplo, analítica de datos con un $2,9 \%$, seguridad informática con el mismo porcentaje de uso, sistemas CRM con un 2,9\%, ERP con un $8,8 \%$, internet de las cosas con un $11,8 \%$ y almacenamiento en nube — cloud computing — con un 35,3\%; este último es el de mayor uso. Se resaltan aspectos como conectividad en el $100 \%$ de los hoteles, con velocidades de navegación mayores a $11 \mathrm{Mb}$.
Se observa un uso creciente en marketing digital con el $47,1 \%$ y una percepción positiva con respecto al incremento en los ingresos por el uso de las TIC, y en el mejoramiento de la competitividad del sector, con un $73,6 \%$ y $85,3 \%$, respectivamente.

Se debe entender que el sector hotelero puede monetizar o sacar provecho comercial y de crecimiento con el manejo adecuado de los datos de información que generan los hoteles. Se sugiere, además, el desarrollo de un programa de fidelización para maximizar el impacto de las campañas de marketing y la incorporación de recursos en analítica de datos y big data.

Es necesario, entonces, que el sector hotelero en Villavicencio, Colombia, maximice su capacidad instalada en términos de infraestructura tecnológica, 
que si bien es aún incipiente en términos de software de gestión, relacionada, de una parte, con la gestión del hotel y, de otra, con las tendencias globales en cuanto a la industria 4.0, debe aprovechar el potencial que se

\section{Referencias}

Alberca, M.; Parte, L. (2010). Nuevas tecnologías y productividad en las empresas hoteleras: evidencia empírica (2000-2005). Turismo y Desarrollo Local, 3(7), 1-20.

Bastakis, C.; Buhalis, B.; Butler; R. (2004). The perception of small and medium sized tourism accommodation providers on the impacts of the tour operators' power in Eastern Mediterranean. Tourism Management, 25(2),151-170. DOI: https://doi.org/10.1016/S0261-5177(03)00098-0

Botello, H.; Pedraza, A.; Contreras, O. (2015). Análisis empresarial de la influencia de las TIC en el desempeño de las empresas de servicios en Colombia. Revista Virtual Universidad Católica del Norte, (45), 3-15.

Bresnahan, T.; Brynjolfsson, E.; Hitt, L. M. (2002). Information technology, workplace organization and the demand for skilled labor: firm level comments firm level evidence. Quarterly Journal of Economics, 117(1), 339-376. DOI: https://doi. org/10.1162/003355302753399526

Buhalis, D.; Law, R. (2008). Progress in information technology and tourism management: 20 years on and 10 years after the Internet. The state of eTourism research. Tourism Management, 29(4), 609-623. DOI: https://doi.org/10.1016/j. tourman.2008.01.005

Camisón, C. (1995). Dirección hotelera y tecnologías de la información: un análisis empírico de la empresa hotelera Valenciana. Estudios Turísticos, (125), 19-52.

Choi, S.; Mattila, A. (2006). The role of disclosure in variable hotel pricing. Cornell Hotel and Restaurant Administration Quarterly, 47(1), 27-35. DOI: https://doi. org/10.1177/0010880405281681

Espín, J. M.; Salazar, C. F.; Jara, M. del R.; García, Y. (2017). Uso de las TIC para la gestión hotelera en el sector turístico de Pastaza. Ventana Informática, (36), 35-52. DOI: https://doi. org/10.30554/ventanainform.36.2385.2017

Fernández-Alcantud, A.; López-Morales, J. M.; MorenoIzquierdo, L.; Perles-Ribes, J. F.; Ramón-Rodríguez, A. B.; Such-Devesa, M.J. (2017). Innovación y destinos inteligentes: oportunidad para el know how turístico español. Información Comercial Española (ICE). Revista de Economía, (894), 137 150.

García-Peñalvo, F. J.; Seoane-Pardo, A. M. (2015). Una revisión actualizada del concepto de eLearning. Education in The Knowledge Society, 16(1), 119-144. DOI: https://doi. org/10.14201/eks2015161119144 tiene en la región en términos de oferta turística, en la cual el sector hotelero es un actor relevante dentro de estas dinámicas sectoriales.

Geyskens, I.; Steenkamp, J.-B.; Kumar, N. (1999). A meta-analysis of satisfaction in marketing channel relationships. (1999). Journal of Marketing Research, 36(2), 223-238. DOI: https:// doi.org/10.2307/3152095

Gobernación del Meta (2009). Plan Regional de Competitividad del Departamento del Meta 2008-2032. Villavicencio: Secretaría de Planeación y Desarrollo Territorial- Gobernación del Meta

Jacob, M.; Aguiló, E. (2008). La innovación en el sector turístico: el caso de Baleares Universitat de les Illes Balears. ROTUR/ Revista de Ocio y Turismo, 1, 51-64. DOI: https://doi. org/10.17979/rotur.2008.1.1.1225

Lindenmeier, J.; Tscheulin, D. K. (2008). The effects of inventory control and denied boarding on customer satisfaction: the case of capacity-based airline revenue management. Tourism Management, 29(1), 32-43. DOI: https://doi.org/10.1016/j. tourman.2007.04.004

López, A.; López, S. (2018). Impacto de las TIC en el turismo: caso colombiano. Cuadernos de Turismo, (41), 399-418. DOI: https://doi.org/10.6018/turismo.41.327081

Martínez, J. (2013). Los sistemas de información en el mundo hotelero: un modelo de éxito (Tesis de doctorado). Universitat de Girona, España. Recuperado de http://hdl.handle. net/10803/319717

Majó, J.; Moya, D.; Vall-Llosera, L. (2018). Impacto de las redes sociales en los ingresos de los hoteles de Colombia, Ecuador y Perú. Revista Facultad de Ciencias Económicas, 26(1),147-162. DOI: https://doi.org/10.18359/rfce.3143

Oliveros, D.; Martínez, G. (2017). Efecto de las TIC sobre la gestión de las empresas hoteleras afiliadas a Cotelco de Bucaramanga (Santander, Colombia). Revista Escuela de Administración de Negocios, (83), 15-30. DOI: https://doi. org/10.21158/01208160.n83.2017.1827

Pablos-Pons, J. de. (2018). Las tecnologías digitales y su impacto en la universidad. Las nuevas mediaciones. RIED. Revista Iberoamericana de Educación a Distancia, 21(2), 83-95. DOI: https://doi.org/10.5944/ried.21.2.20733

Padilla, A.; Garrido, A. (2012). Gestión de relaciones con clientes como iniciativa estratégica: implementación en hoteles. Revista Venezolana de Gerencia, 17(60), 587-610.DOI: http:// dx.doi.org/10.37960/revista.v17i60.10934 
Palmer, A.; McCole, P. (2000). The role of electronic commerce in creating virtual tourism destination marketing organisations. International Journal of Contemporary Hospitality Management, 12(2,3), 198-204. DOI: https://doi. org/10.1108/09596110010320760

Ruiz, M.; Gil, I.; Moliner, B. (2012). Tecnologías de la información en el sector hotelero y sus implicaciones en las relaciones empresa-cliente. Revista de Análisis Turístico, (13), 11-26.

Santomá, R. (2004). Comparación en el Uso del E-mail y del equipamiento en Tic entre Hoteles de Barcelona, París, Londres, Berlín y Roma. Ponencia presentada en el V Congreso Turismo y Tecnologóia de la Información y la Comunicación - Turictec 2004.

Stamboulis, Y.; Skayannis, P. (2003). Innovation strategies and technology for experience-based tourism. Tourism Management, 24,35-43. DOI: https://doi.org/10.1016/ S0261-5177(02)00047-X

Schwab, K. (2016). La cuarta revolución industrial. Barcelona: Debate. 